\title{
Reducing turnaround time of surgical pathology reports in pathology and laboratory medicine departments
}

\author{
Saeed Alshieban, Khaled Al-Surimi \\ King Abdulaziz Medical City, King Saud bin Abdulaziz University for Health Sciences, Riyadh, Kingdom of Saudi Arabia
}

\begin{abstract}
Turnaround time is an important quality indicator in surgical pathology. Retrospective analysis of three data points in September 2014, January 2015 , and February 2015 showed that on average, about a quarter (24\%) of routine surgical pathology cases $(26 \%$, $19 \%$, and $27 \%$ respectively) are not reported on time and do not meet the accepted level of the College of American Pathologists' (CAP) standard turnaround time, which states at least $90 \%$ of routine surgical pathology cases should be reported and verified within two days. Our daily observation supported by a root cause analysis exercise revealed that subprocesses including slide allocation and delivery to pathologists, slide review by pathologists, report editing by transcriptionists, and report verification by pathologists are where most delays occur. Thus, to tackle these process issues we developed a quality improvement project using the model of improvement methods to streamline the sample flow process and avoid unjustified reporting delay. The improvement ideas included developing a time log sheet to be attached with all pathology requests, and sending a daily reminder email followed by a phonecall to all pathologists to verify completed reports on the same day. These intervention elements were tested using multiple PDSA cycles resulting in a very noticeable improvement, with more than $94 \%$ of all routine cases reported in less than two days, meeting and exceeding the CAP standards. Such noticeable improvement in turnaround of surgical pathology reports will eventually lead to improving the quality and safety of patient care outcome, including diagnosing patients on time, developing the appropriate treatment plan, and avoiding unjustified complications resulting in morbidity and mortality due to delayed reports.
\end{abstract}

\section{Problem}

Turnaround time is a major quality indicator for surgical pathology laboratories. According to the College of American Pathologists (CAP), most routine cases (>=90\%) should have a turnaround time of two days or less for the surgical pathology report. However, a retrospective analysis of three data points in September 2014, January 2015, and February 2015 showed, on average, 24\% (26\%, $19 \%$, and $27 \%$ respectively) of the surgical pathology reports had not been submitted on time, and fell outside the CAP standard approved time (see figure 1 and figure 2). The implication of surgical pathology report delay as shown in previous research evidence is that prolonged turnaround time plays a major role in disease complications, including raising morbidity and mortality rates. Thus, we decided to employ quality improvement methods and tools to reduce the turnaround time of surgical pathology laboratory reports, and meet the CAP standard approved turnaround time.

\section{Background}

Timely anatomical pathology reports are one of the most important tools physicians use to adequately manage the quality and safety of patient care.[1] Hence, verifying pathology reports in an appropriate time frame helps health care practitioners with diagnosing patients in a timely fashion, which will lead to an effective treatment plan.[2] Research evidence shows that a prolonged turnaround time raises morbidity and mortality rates.[3] Most pathology departments worldwide follow the standards of one of the known international authorities, such as The Joint Commission (TJC), CAP, and others.[4,5] Our surgical pathology laboratory department follows the CAP standards, which requires providing precise evaluation and reporting of specimens for patient management plans. Thus, application of quality assurance systems within our department is a priority, using many quality indicators including for example turnaround time. The accuracy of diagnosis and providing timely complete reports is one of the main quality indicators in surgical pathology.[6] Turnaround time is considered the key daily quality performance evaluation element due to several reasons: firstly, it can be assessed easily with laboratory information systems; secondly, it has a strong economic impact on cost effectiveness; and thirdly, it is part of the equation of physician satisfaction indicators. $[1,6,7]$ In summary, to meet the CAP standards requires the turnaround time of surgical pathology reports for $90 \%$ of routine cases to be reported within two days or less, to improve quality outcomes of treatment plans.[8]

\section{Baseline measurement}

For the purpose of this quality improvement project a retrospective analysis of turnaround times was collated for all surgical pathology reports submitted during the months of September 2014, January 2015, and February 2015, from the time samples reached the pathology laboratory until they were verified by the pathologist. The data was extracted from the electronic laboratory information system. The time was labeled as day zero for cases verified on the day of receipt, day one for cases verified the next day, day two for cases verified two days later, etc. The findings of the data showed about a quarter $(24 \%)$ of surgical pathology cases were reported beyond the two day turnaround time as required by CAP standards (see figure 1, figure 2). 
See supplementary file: ds6579.pptx - "\% of TATs for surgical pathology reports beyond two days, as required by CAP standards; sample process flow chart; log sheet"

\section{Design}

By looking at sample process flow charts within the pathological laboratory, our first observation was that the time needed for the sample flow to move from one process to another was not registered, hence the delay is not noticeable from within the flow process itself. Moreover, our observation experience and root cause analysis exercise revealed that the processes of slide allocation and delivery to pathologists, slide review by pathologists, report editing by transcriptionists, and report verification by pathologists are the subprocesses where most delays occur. After analysis of this problem using a cause effect diagram followed by a Pareto chart and prioritisation matrix, we concluded late slide handover to pathologists to be a target area for our improvement project (see figure 3: sample process flow chart). As a result, a time log sheet was created and attached to the front sheet of the pathology request form, recording the time spent in every process, to ensure each subprocess was completed on time (see figure 4). In addition, daily email and mobile phone messages were sent to all pathologists to remind them to verify completed reports before the end of the day.

\section{Strategy}

Before starting the quality improvement cycles the change ideas were highlighted to the relevant staff in the surgical pathology division within the laboratory department, including pathologists, physician representatives, transcriptionists, laboratory quality officers, laboratory directors, laboratory technologists, and administration assistants.

\section{PDSA cycle 1}

We started by attaching a time log sheet to all surgical pathology request forms. The time for each sample processing stage was documented by medical technologists and administration assistants until the slides reached the pathologist's office. Then the pathologist took over to document the time spent in the main process until verification. Transcriptionists then entered all data into an Excel worksheet and calculated the turnaround time. In addition to the time log sheet, every day an email and mobile phone reminder message was sent to all pathologists to verify pending cases. The change process went smoothly, however on the second day one pathologist had a problem logging in to her computer that took an additional day to fix.

PDSA cycle 2

This cycle was to fix the login problem for the affected pathologist's computer and all pathologists' computers; the problem had been identified as a result of testing PDSA cycle 1.

PDSA cycle 3
We carried out the project improvement ideas for 10 consecutive days, and noticed the improvement idea started working very well, that there was a clear and noticeable improvement in reducing delays to the turnaround time of surgical pathology reports, and meeting and exceeding CAP standards regarding surgical pathology reports.

After this PDSA cycle we decided to assess the satisfaction of the surgical pathology laboratory staff about the project. We found they were satisfied with the project idea and results, and expressed their full support for the project idea and its sustainability in the future.

\section{Results}

During the period of testing a total of 168 surgical pathology reports were recorded. Analysis following the project showed a noticeable decrease in the data median from the first day of testing the change, from 53.5 hours to 41 hours. The data was interrupted on the second day due to a computer login problem encountered by one pathologist; however, this special course was fixed in the system and improvement progress went back to initial improvement levels after solving the issue (see figure 5 : the process run chart). Testing of the change ideas went smoothly and the turnaround time showed good improvement, with $94 \%$ of all routine cases reported within less than two days, meeting CAP standards (see figure 6). Moreover, all staff involved in the process of surgical reporting were satisfied with the change process and noticeable improvements.

See supplementary file: ds6580.pptx - "Process run chart and turnaround time for surgical pathology reports before and after improvement project"

\section{Lessons and limitations}

We learnt several lessons from this improvement project. One important lesson we learnt is that small and simple change can lead to huge improvements; it is not necessary that improvement needs big and complex changes. In addition, we noticed engaging people and improving communication skills and teamwork among colleagues made the improvement ideas easy to be implemented, and helped in exploring the potential problems and obstacles that might occur during the improvement journey. An important limitation to this project is the small sample number (168 surgical pathology reports) which may have had an impact on the outcome of the study; however, the general idea was to come up with a tool to improve the turnaround time. We believe that the change idea tested is easy and practical to be used.

\section{Conclusion}

This project improvement idea of mapping out the workflow process has led to process and systems improvement. Focusing on fixing subprocesses within the sample flow process had led to more than $94 \%$ of all routine surgical pathology cases being reported within less than two days and meeting the CAP standards. We think such improvements will lead to improving quality and patient safety, including timely diagnoses, improving patient treatment plans, and 
avoiding any unjustified disease complications such as morbidity and mortality due to reporting delay. Hence, it is clear that mapping out all process flows within patients' hospital interventions is an important quality tool and can help in identifying potential areas with quality problems or defects, and lead to new improvement projects.

\section{References}

1. Patel S, Smith JB, Kurbatova E, Guarner J. Factors that impact turnaround time of surgical pathology specimens in an academic institution. Hum Pathol 2012;43(9):1501-5.

2. Nakhleh, RE. Introduction. In: Nakhleh RE, Fitzgibbons PL, editors. Quality Management in Anatomic Pathology: Promoting Patient Safety Through Systems Improvement and Error Reduction. Northfield (IL): The College of American Pathologists, 2005. p.1-4.

3. Jerjes W, Upile T, Radhi H, et al. Delay in pathological tissue processing time vs. mortality in oral cancer: Short communication. Head Neck Oncol 2012;4:14.

4. Röcken C, Manke H. Accreditation in pathology. Systematic presentation and documentation of activities in pathology. Pathologe 2010;31(4):268-78.

5. Nakhleh RE. What is quality in surgical pathology?. J Clin Pathol 2006; 59(7): 669-672.

6. Vollmer RT. Analysis of turnaround times in pathology: an approach using failure time analysis. Am J Clin Pathol 2006;126(2):215-20.

7. Nakhleh RE, Souers R, Ruby SG. Physician satisfaction with surgical pathology reports: a 2-year College of American Pathologists Q-Tracks Study. Arch Pathol Lab Med 2008;132(11):1719-22.

8. Novis DA, Zarbo RJ, Saladino AJ. Interinstitutional comparison of surgical biopsy diagnosis turnaround time: a College of American Pathologists Q-Probes study of 5384 surgical biopsies in 157 small hospitals. Arch Pathol Lab Med 1998;122(11):951-6.

\section{Declaration of interests}

Nothing to declare

\section{Acknowledgements}

Dr Mohammad Ashraf Aziz, Dr Awad Alshahrani, Mr Hasan Alnakhli, Ms Rica Ocular, Mr Dave Cuasay

\section{Ethical approval}

The work of this quality improvement project is primarily intended to improve quality performance of pathology and laboratory medicine departments, and not to generate new generalisable knowledge about performance. Thus, the department of pathology and laboratory medicine fully supported the project idea to be implemented, so there was no need for IRB approval to conduct this quality improvement project. 